\title{
Thymic tumours: a single center surgical experience and literature review on the current diagnosis and management of thymic malignancies
}

\author{
Fabrizio Minervini ${ }^{1} \wedge$, Laura Boschetti ${ }^{2} \wedge$, Michael Gregor $^{2} \wedge$, Mariano Provencio $^{3} \wedge$, Virginia Calvo $^{3} \wedge$, \\ Peter B. Kestenholz ${ }^{1} \wedge$, Savvas Lampridis ${ }^{4} \wedge$, Davide Patrini ${ }^{5} \wedge$, Pietro Bertoglio $^{6} \wedge$, L. Filipe Azenha ${ }^{1} \wedge$, \\ Consolato M. Sergi ${ }^{7} \wedge$, Gregor J. Kocher ${ }^{8 \wedge}$
}

${ }^{1}$ Department of Thoracic Surgery, Cantonal Hospital Lucerne, Lucerne, Switzerland; ${ }^{2}$ Department of Hematology, Cantonal Hospital Lucerne, Lucerne, Switzerland; ${ }^{3}$ Department of Medical Oncology, Hospital Universitario Puerta de Hierro-Majadahonda, Madrid, Spain; ${ }^{4}$ Department of Thoracic Surgery, 424 General Military Hospital, Thessaloniki, Greece; ${ }^{5}$ Department of Thoracic Surgery, University College London Hospitals, London, UK; ' ${ }^{6}$ ivision of Thoracic Surgery, IRCSS Azienda Ospedaliero-Universitaria, Bologna, Italy; ${ }^{7}$ Division of Anatomic Pathology, Children's Hospital of Eastern Ontario, University of Ottawa, Ontario, Canada; ${ }^{8}$ Division of Thoracic Surgery, Bern University Hospital, University of Bern, Bern, Switzerland

Contributions: (I) Conception and design: F Minervini; (II) Administrative support: None; (III) Provision of study materials or patients: All authors; (IV) Collection and assembly of data: All authors; (V) Data analysis and interpretation: All authors; (VI) Manuscript writing: All authors; (VII) Final approval of manuscript: All authors.

Correspondence to: Fabrizio Minervini, MD, PhD. Department of Thoracic Surgery, Cantonal Hospital Lucerne, Lucerne, Switzerland.

Email: fabriziominervini@hotmail.com.

Objective: This study aimed to provide an extensive overview of clinical and pathological findings along with various therapeutic options analyzing in addiction, retrospectively, the surgical outcomes of a single center cohort.

Background: Thymic neoplasms are rare thoracic tumors which commonly are located in the anterior mediastinum and are associated with a wide spectrum of clinical presentations. They may run an indolent course or could present a very aggressive biologic progression with infiltration of mediastinal structures and presence of distant metastases. The pathogenesis of these tumors is so far not completely clear. Several treatment modalities in a multidisciplinary setting have to be considered in order to provide the best treatment for patients affected by thymic tumors.

Methods: We conducted a retrospective cohort analysis of all patients who underwent surgery due to thymic tumor in a university hospital located in Switzerland (Bern University Hospital) and then we performed a narrative review of the English literature using PubMed, Embase, Cochrane Database of Systematic Reviews and Scopus.

Conclusions: Minimally invasive techniques play an important role in the treatment of thymic tumors. A careful patients selection in a multidisciplinary setting is mandatory in order to offer the best treatment for patients affected by thymic tumors.

Keywords: Thymoma; thymic tumors; thymic carcinoma

Submitted Aug 04, 2021. Accepted for publication Oct 07, 2021.

doi: $10.21037 / g s-21-517$

View this article at: https://dx.doi.org/10.21037/gs-21-517

^ ORCID: Fabrizio Minervini, 0000-0003-3595-7307; Laura Boschetti, 0000-0002-8524-8077; Michael Gregor, 0000-0002-2322-4897; Mariano Provencio, 0000-0002-2826-656X; Virginia Calvo, 0000-0002-3503-4847; Peter B. Kestenholz, 0000-0002-0419-3685; Savvas Lampridis, 0000-0003-2827-5826; Davide Patrini, 0000-0002-2492-799X; Pietro Bertoglio, 0000-0002-6267-0099; L. Filipe Azenha, 00000003-2883-5205; Consolato M. Sergi, 0000-0002-2779-7879; Gregor J. Kocher, 0000-0001-6531-2919. 


\section{Introduction}

Thymic tumors are rare neoplasms that arise from the anterior mediastinum. Characteristic feature of these tumors is the clinical and pathologic wide spectrum ranging from well encapsulated tumors with a benign course to highly invasive and aggressive ones. They usually occur in the fourth to sixth decade of life accounting for $0.2-1.6 \%$ of all malignant tumors (1-3).

The classification of thymic tumors has represented a big challenge and indeed different pathologic classifications have been developed and published over the years (2).

In 1981, Masaoka et al. published a new classification to assign the patients to one of four clinical stages (4). A later adaptation made by Koga et al. in 1994 resulted in the widely accepted Masaoka-Koga staging system (5).

The 8th edition of the TNM classification of malignant tumors published in 2017 included for the first time an official staging system for thymic tumors based on a proposal of the International Association for the Study of Lung Cancer (IASLC) and the International Thymic Malignancy Interest Group (ITMIG) $(6,7)$.

However, the Masaoka-Koga classification along with the WHO histologic classification are the most widely used classification systems for thymic tumors, representing a useful reference for surgeons, oncologists and radiation oncologists (8).

The treatment's cornerstone of thymic tumors is the patients selection, based on a multidisciplinary approach.

For patients who are suitable for surgery, an important prognostic factor is the radicality.

For years, an open surgical approach was considered the gold standard but nowadays several minimal invasive approaches can be used by experienced surgeon with good outcomes.

We present the following article in accordance with the Narrative Review reporting checklist (available at https:// dx.doi.org/10.21037/gs-21-517).

\section{Methods}

We conducted a retrospective cohort analysis of all patients who underwent surgery due to thymic tumor. We analyzed clinical and patient outcomes. The retrospective analysis was approved by institutional board and individual consent was waived.

In the discussion session we conducted a narrative literature review using PubMed, Embase, Cochrane
Database of Systematic Reviews and Scopus. Studies examining thymic tumors were identified combining the terms: "thymus", "thymic tumors", "thymoma" as well as "thymic malignancies" as either keywords or MeSH terms for articles in English language. Relevant papers and linked articles published between 1970 and 2021 were included and screened. Due to the narrative design of the review a certain subjectivity in choice of studies included is likely.

\section{Statistical analysis}

Continuous variables are reported as mean in normally distributed data; discrete variables are reported as numbers and percentage. All data were de-identified with a sequentially-generated study identification code, encrypted, and transferred to the central Lucerne site for analysis. SPSS 25.0 (www.ibm.com) was used for statistical analysis.

\section{Results}

\section{Single center demographics and outcomes}

The single center experience of the thoracic surgery department of the University of Bern, Switzerland, confirms the feasibility and safety of minimally invasive techniques. Out of a total of 104 resections for anterior mediastinal tumors performed between January 2010 and December $2020,77.9 \% \quad(n=81)$ were done by minimally invasive surgery and all of these procedures were done using the daVinci robotic platform. Tumor resection via median sternotomy was performed in 22 patients $(21.1 \%)$ and mainly for thymic carcinoma $(\mathrm{n}=3 ; 2.86 \%)$ or large thymomas $(\mathrm{n}=17$; size range, $4.8-23 \mathrm{~cm})$. Hemiclamshell was performed in 1 patient (1\%) with a large tumor invading the right hemithorax. Histology showed benign lesions in $41.3 \%(n=43)$, thymoma in $51 \%(n=53)$, and thymic carcinoma in $3.8 \%(n=4)$ of patients. Additionally, histology revealed a teratoma in $2.9 \%(\mathrm{n}=3)$ and lymphoma in $1 \%(\mathrm{n}=1)$ of patients. The daVinci platform was used for tumors with a size ranging from 1.5 to $9 \mathrm{~cm}$ and median LOS of these patients was 3.2 days (range, 2-12 days). On the other hand, mean LOS of patients operated using transsternal approach was 9.9 days (range, 4-33 days). The last group had a higher rate of postoperative complications requiring therapy, $4.8 \%(\mathrm{n}=5)$ for minimally invasive $v s$. $9.6 \%(\mathrm{n}=10)$ for transsternal.

The main complications included bleeding $(\mathrm{n}=2)$, 
pneumonia $(\mathrm{n}=2)$, chylothorax $(\mathrm{n}=1)$; myasthenic crisis $(n=1)$, pericardial effusion $(n=2)$, intestinal perforation $(n=2)$, surgical site infection $(\mathrm{n}=2)$, urosepsis $(\mathrm{n}=1)$ and phrenic nerve paralysis $(\mathrm{n}=2)$.

In patients with thymoma, Masaoka tumor stages were I in 22 patients (42\%), IIa in 19 patients (35.6\%), IIb in 7 patients (13.2\%), III in 3 patients $(5.6 \%)$ and IVa in 2 patients (3.6\%).

After a mean follow-up of 44 months (range, 1108 months), $\mathrm{n}=4$ patients with thymoma showed recurrence, 3 were treated via a transsternal approach and only 1 was treated using the daVinci platform. In the group with thymic carcinoma 1 patient showed pleural recurrence after 14 months and was treated by minimally invasive resection of the recurrence sites.

Five patients have died during follow-up, only 1 of them due to tumor progression.

\section{Discussion}

\section{Clinical findings}

Most thymic tumors, just like several mediastinal masses, are asymptomatic and are discovered as incidental findings in chest radiography or computed tomography (CT). Furthermore, in the specific, a CT scan with IV contrast provides essential information about the size, location, tissue consistency and involvement of other structures.

PET/CT is not considered a standard in the diagnostic pathways except to rule out extrathoracic metastases even though if some authors consider it very useful as diagnostic tool (9-11). It's generally agreed that benign tumors are more often asymptomatic than malignant counterparts (12). The most common symptoms, although nonspecific, are chest pain, cough and dyspnea. If the tumor invaded or compressed adjacent organs or structures could cause several symptoms like stridor, dysphagia, superior vena cava syndrome, hoarseness or hemoptysis.

Pleural or pericardial effusions as well as spontaneous tumor rupture have been rarely observed $(13,14)$.

\section{Pathology}

Thymic tumours have been traditionally a difficult topic for both residents and staff. The classification of this kind of tumors has been challenged by the mixture of epithelial and hematologic components that involves two different embryological germ layers in the embryology (ectoderm and mesoderm). The 4th edition [2015], now recently revised [2021] classification of thymic tumors comprises categories based on what the tumor cells are identified under a microscope. Three Latin letters (A, B, and C) are used with some subtyping and combination. In type A thymoma, which is also known as spindle cell thymoma or medullary thymoma. There is a spindle cell morphology, which is quite different from the round or ovoidal cytology found in the B and C neoplasms. There is a good recovery for people with type A thymoma and nearly $100 \%$ of people with this type live at least 15 years after diagnosis.

The atypical type A thymoma variant was introduced in the 4th edition of the classification of thymic tumors. This variant shows hypercellularity, increased mitotic activity, and evidence of necrosis. Type B thymoma is characterized by a bioreactivity and looks like thymus of fetal and infantile appearance. There are three subcategories, including B1 through B3. The B1 subtype is also known as lymphocyte rich and looks like normal functional thymus. It demonstrates combining large areas having normal thymic cortical areas with areas showing thymic medulla.

Substantially, in B1 there is a thymus-like architecture and cytology with copious immature $\mathrm{T}$ cells, areas of medullary differentiation (the so-called "medullary islands"), and scattered polygonal or dendritic epithelial cells without clustering (i.e., $<3$ contiguous epithelial cells).

In the B2 subtype, or cortical type, the neoplastic epithelial component is highlighted as scattered plump cells. They are identified harbouring vesicular nuclei and distinct nucleoli. There is impressive component of lymphocytes. Perivascular spaces are quite common to be identified. Substantially, increased single or clustered polygonal cells or dendritic epithelial cells intermingled with immature $\mathrm{T}$ cells are seen. Finally, the B3 subtype or lymphocytepoor subtype, shows epithelial cells with round/polygonal cytology. Mild atypia is encountered. Atypia is mixed with a minor component of lymphocytes. At places, foci of squamous metaplasia and perivascular spaces are identified. Substantially, in B3 there are sheets of polygonal slightly to moderately atypical epithelial cells with rare or absent intercellular bridges, and scattered or no intermingled $\mathrm{Td} \mathrm{T}+\mathrm{T}$ cells.

Thymic carcinoma are unencapsulated tumors with no internal fibrous septation and characterized histologically as cohesive cells with regular round or oval nuclear outlines, eosinophilic prominent nucleoli, and geographic necrosis. There are commonly foci of medullary differentiation, some aberrant or abortive Hassall corpuscles, rosettes, 
and gland-like spaces, without perivascular spaces. In the keratinizing variant intercellular bridges and keratin pearls are seen. In the non-keratinizing variant angular nests of atypical squamous cells in desmoplastic stroma are identified. There is no evidence of intercellular bridges, eosinophilic cytoplasm, or keratin pearls in the nonkeratinizing variant.

The immunohistochemistry plays a major role in the classification of thymic tumors. Cytokeratins, or keratins as they are now labelled, are intermediate filaments of the cytoskeleton and are found in epithelial cells of normal thymus, thymomas $\mathrm{A}$, thymic carcinomas, neuroendocrine tumors, germ cell tumors, dendritic cell tumors, and very rarely sarcomas. Also they can be seen in metastases to the mediastinum. The keratin 19 is seen in epithelial cells of normal thymus, thymomas $\mathrm{A}$, and thymic carcinomas. The keratin 20 is seen in normal thymus and thymomas. Also it is positive in rare thymic adenocarcinomas, teratomas, or, even, metastases. P63 or tumor protein p63 is a member of the p53 family of transcription factors. In mice knocked out for p63 (p63-/- mice) there are several developmental defects which include the lack of limbs and other tissues, such as mammary glands and teeth. The antigen P63 is found in the nuclei of normal and neoplastic thymic epithelial cells, primary mediastinal large B-cell lymphoma, and, of course, squamous epithelial cells (e.g., in teratoma, metastasis), P40 is an isoform of P63. The antibody p40, which identifies $\Delta \mathrm{Np} 63$, has been available for numerous years but its use for distinction of squamous cell carcinoma of the lung and adenocarcinoma has been only recently highlighted. P40 is consistently expressed in squamous cell carcinoma. In thymus, $\mathrm{P} 40$ is positive in the nuclei of normal and neoplastic thymic epithelial cells, and squamous epithelial cells (e.g., in teratoma, metastasis). TdT or terminal deoxynucleotidyl transferase is a nuclear DNA polymerase present in thymic and small number of bone marrow cortical lymphocytes. TdT determines the catalysis of the addition of deoxynucleotides to 3' $\mathrm{OH}$ ends of oligonucleotides or polydeoxynucleotides, carries out DNA synthesis using only single-stranded DNA as the nucleic acid substrate, and is absent in fetal liver hematopoietic stem cells. TdT is the classic (nuclear stain) marker of B or T cell acute lymphoblastic leukemia/lymphoma. TdT is present in the immature T cells of normal thymus, almost all $(>90 \%)$ of thymomas, and neoplastic $\mathrm{T}$ cells of $\mathrm{T}$ lymphoblastic lymphoma. The cluster differentiation 5 or CD5 is a marker for Immature and mature $\mathrm{T}$ cells of thymus and is present in more than $90 \%$ of thymomas, neoplastic $\mathrm{T}$ cells of many
T lymphoblastic lymphomas, as well as epithelial cells in about two-thirds of thymic carcinomas. The B lymphocyte marker, CD20 is present in normal and neoplastic B cells, epithelial cells in $50 \%$ of cases of type A thymoma, and about $50 \%$ of the $\mathrm{AB}$ thymoma. Finally, c-kit or CD117 is found in the epithelial cells in $80 \%$ of thymic carcinomas, as well as neoplastic cells in most seminomas, which can occur in the mediastinum.

The differential diagnosis of thymomas includes Burkitt lymphoma, which shows obviously a different nuclear histology, Castleman disease, which is usually not located in thymus, "onion skinning" pattern by lymphocytes, some fibrohyaline or plasma cell subtypes, and lymphoblastic lymphoma, which harbors similar staining as thymomas but they are negative for keratin. Thus, it is important to stain for keratins for tumors of the mediastinum even the flow cytometry is indicative of lymphoblastic lymphoma. Also, it is important not to over- or misinterpret as thymomas some lymphoblastic lymphomas with scattered positive staining of trapped epithelial cells. Thymic lymphoid hyperplasia is characterized by normal cortical and medullary glandular distinction, which is maintained, well-formed germinal centers, and there is no evidence of a tumor-forming mass (15).

There are numerous genetic, transcriptomic, and epigenetic studies that have addressed the distinction of thymic carcinomas from thymomas. Thymic carcinomas harbor mutations of expression profiles of anti-apoptotic genes, methylation patterns, and epigenetic regulatory genes that distinguish them from thymomas. The identification of point mutations in the GTF2I oncogene in all major thymoma subtypes and thymic carcinomas characterize this cohort of thoracic tumors. GTF2I oncogene is a protein that in humans is encoded by the GTF2I gene. This gene encodes a phosphoprotein with roles in transcription and signal transduction and is deleted in Williams-Beuren syndrome, a multisystem developmental disorder caused by the deletion of contiguous genes at chromosome 7q11.23.

Thymic epithelial tumors (TETs) frequently associate paraneoplastic syndromes:

(I) Myasthenia gravis (MG);

(II) Pure autoimmune red cell aplasia;

(III) Hypogammaglobulinemia.

\section{$M G$}

One of the most common paraneoplastic disease associated 
with thymic abnormalities is MG. About $70-80 \%$ of patients with MG have thymic hyperplasia or thymoma but, even rare, few cases of thymus carcinoma have been reported $(16,17)$.

MG is a heterogeneous neuromuscular disease caused by an autoimmune mediated response against post synaptic nicotinic acetylcholine receptors (AChRs) preventing their activation at the neuromuscular junction.

The exact pathophysiologic mechanisms that trigger the AChRs antibodies production are not clear so far. Probably in some patients, auto-reactive $\mathrm{T}$ cells can recruit AChR-responsive B cells, stimulating the production of pathogenic anti AChR antibodies. As consequence, the decreased number of functional receptors causes a reduced neuromuscular transmission (18). The primum movens of the $\mathrm{T}$ cells dysregulation remains unclear, although Epstein-Barr virus infection has been postulated (19).

Twenty percent of patients with MG present only antibodies against muscle specific tyrosine kinase (MuSK), a subset of immunoglobulin G4. Up to $50 \%$ of patients with AChR and MuSK negative antibodies present antilow-density lipoprotein receptor-related protein 4 (Lrp4) antibodies, an immunoglobulin G1 subclass which inhibits the binding of their target with agrin, a protein which plays a central role in the function of the neuromuscular junction (2).

MG is clinically characterized by a pattern of weakness with a fluctuating course leading to period of exacerbation and period of remission. In most cases, weakness involves extraocular muscles (ptosis, double vision), bulbar muscles (impaired chewing, swallowing and speaking), facial and respiratory muscles (breathlessness up to ventilatory insufficiency) or extremity muscles.

A dreaded complication of MG is the myasthenic crisis, often requiring admission to an intensive care unit and ventilatory support but despite that it could bring along a significant mortality.

The diagnosis of MG is based on the detection of myasthenic antibodies in the serum along with electrophysiological tests such as single-fiber electromyography (SFEMG) and by repetitive nerve stimulation (RNS). Inhibition of acetylcholinesterase (AChE) with pyridostigmine or ice pack test, which normally ameliorates the symptoms, have been routinely applied.

Next diagnostic step after the diagnosis of MG, should be a CT scan of the chest in order to detect a possible thymic hyperplasia or tumors. In case suspected thymic mass, a biopsy should be avoided due to the risk of exacerbating the disease.

\section{Other paraneoplastic diseases}

Paraneoplastic syndromes may precede the presentation of a thymic tumor, can be diagnosed together with it, or may occur after treatment (with or without evidence of tumor recurrence). The disrupted thymic architecture as well as the decreased or lacking expression of autoimmune regulator (AIRE) and major histocompatibility complex (MHC) Class II, leading to an abnormal T-cell maturation, is thought to be the pathogenetic mechanism beyond the development of the thymic autoimmune-mediated paraneoplastic disorders.

The epidemiology of paraneoplastic syndromes associated with thymic tumors varies $\mathrm{n}$ the literature. While MG is the most common one, a wide range of other autoimmune paraneoplastic diseases has been reported. Among them common findings are pure red cell aplasia (PRCA) and hypogammaglobulinemia $(20,21)$.

PRCA is an isolated hyporegenerative, normochromic, normocytic anemia in the presence of normal white cell and platelet counts due to a selective autoimmunemediated aplasia of erythroblasts in otherwise normal bone marrow (22). This paraneoplastic disorder occurs in 5\% to $7 \%$ of patients with thymic tumors and is more common in older women (23).

Hypogammaglobulinemia is found in ca $5-20 \%$ of patients with thymic tumors (20). When associated with thymoma it is also designated as Good's Syndrome and the clinical manifestation is typically an adult-onset immunodeficiency causing susceptibility to bacterial, viral, fungal and other opportunistic infections. Good's Syndrome includes not just hypogammaglobulinemia, but also a reduction of peripheral B-cells, $\mathrm{CD} 4^{+}$lymphopenia with reversed $\mathrm{CD} 4 / \mathrm{CD} 8$ ratio. It might have a worse prognosis and a higher mortality than most other immunodeficiencies in adults (e.g., CVID) (24).

Several other paraneoplastic syndromes have been described in the context of thymic tumors related to cardiovascular, neurological, gastrointestinal, endocrine, dermatological, or systemic disorders. Even though not one of the most common, peculiar for thymic tumors in particular for thymomas is the syndrome of thymoma-associated multiorgan autoimmunity 
(TAMA). This disorder, similar to a graft-versus-host disease (GVHD), is T-cell mediated and involves mostly skin, gastrointestinal tract and thyroid gland. Typical clinical manifestations are morbilliform skin eruption, erythroderma and mucosal erosions, chronic diarrhea and liver enzyme alterations as well as autoimmune thyroiditis (23-26).

\section{Surgical management}

\section{Indications for thymectomy}

Thymectomy (i.e., surgical resection of the thymus gland) is generally indicated for the treatment of patients with thymic tumors or nonthymomatous MG. Thymic tumors primarily include thymoma, thymic carcinoma, and thymic neuroendocrine (carcinoid) tumors. Thymic cysts are benign lesions and only require resection if causing symptoms, usually due to compression of adjacent structures, or if imaging reveals a solid component suspicious of malignancy. Similarly, thymic hyperplasia only requires resection in the presence of paraneoplastic symptoms. Regarding the surgical management of nonthymomatous MG, thymectomy is usually reserved for patients who are less than 60 years of age and have either anti-acetylcholinereceptor antibodies or seronegative MG.

Preoperative imaging assessment is paramount to determine potential resectability of thymic tumors. This is typically performed with a computed tomography (CT) of the chest with contrast, which can demonstrate the characteristics of thymic tumors and extent of infiltration of surrounding structures. In this context, it is important to determine if there is an intact thymic capsule or if the tumor extends into mediastinal fat or pleura. Furthermore, it is critical to assess the fat planes separating the tumor from major intrathoracic structures, as disruption of normal tissue planes may suggest infiltration of these structures.

It is worth noting that on particular occasions, surgical resection of anterior mediastinal masses without pre-operative tissue diagnosis is justified to prevent biopsyassociated tumor seeding.

The typical case is the adult patient with a resectable tumor and without other imaging abnormalities or systemic symptoms of lymphoma. In such cases, the potential risk of resecting a lymphoma is favored over the likelihood of worsening the prognosis of a thymoma.

There are currently no consensus guidelines for indicating whether a thymic tumor is unresectable. In a retrospective review of CT scans of 133 patients $(60.2 \%$ with
Masaoka stage I or II thymoma and 39.8\% with Masaoka stage III or IV thymoma) who underwent thymectomy, $17.3 \%$ had an incomplete surgical resection (27). The preoperative CT characteristics that significantly correlated with an incomplete surgical resection included a lobulated tumor contour, greater than or equal to $50 \%$ abutment of the circumference of an adjacent vessel, thoracic lymphadenopathy, adjacent lung changes and pleural nodularity. The mean tumor size was significantly larger in the incompletely compared to the completely resected groups $(9.7$ vs. $6.9 \mathrm{~cm})$. Another retrospective review of CT scans of 99 patients with surgically treated thymoma showed that tumor size greater than or equal to $7 \mathrm{~cm}$, lobulated tumor contour, and infiltration of surrounding fat were significantly associated with Masaoka stage III or IV disease, thereby indicating potential benefit from neoadjuvant therapy for tumors with such characteristics (28).

\section{Surgical techniques}

After a thymic tumor has been deemed amenable to complete resection, the thoracic surgeon must decide the optimal approach. This is usually dictated by the extent of the tumor, invasion of nearby structures, previous thoracic surgery or radiation therapy, patient preferences, and local expertise. Three basic approaches have been widely used for thymectomy: transsternal, transcervical, and minimally invasive.

Transsternal thymectomy is performed via a median sternotomy. It has been considered the standard approach for thymectomy because it provides excellent exposure of the anterior mediastinum, including the thymus, and allows exploration of the neck for complete removal of potentially ectopic thymic tissue. Transsternal thymectomy can also be combined with a transcervical approach.

Transcervical thymectomy is a less radical surgical approach that involves a transverse curvilinear neck incision. This approach has been associated with smaller scars, avoidance of thoracostomy tubes, less postoperative pain, and shorter hospitalizations $(29,30)$. However, it is unsuitable for patients who cannot extend their necks. Reported rates of conversion to a transsternal or thoracoscopic approach range from $0 \%$ to $19 \%$ (30-32). Although it has been argued that the transcervical approach may not expose the thymus in its entirety, thus increasing the risk of incomplete resection, several studies have reported comparable clinical results with transsternal 
thymectomy for patients with MG (30-34).

Minimally invasive thymectomy, which includes video- and robotic-assisted thoracoscopic surgery, can be performed through small incisions for port placement on the right or left side of the chest. Recently, subxiphoid uniportal video-assisted thoracoscopic thymectomy has been also reported by an increasing number of authors $(35,36)$. Robotic-assisted thoracoscopic surgery has the advantage of enhanced vision with three-dimensional or magnified views, reduced tissue trauma (due to use of smaller-diameter instruments and reduced levering against ribs), and improved dissection in small spaces. However, it lacks haptic feedback for the surgeon, a feature that is provided by video-assisted thoracoscopic surgery (37). Minimally invasive techniques are usually reserved for stage I or II thymomas, thymic carcinomas less than $3 \mathrm{~cm}$ in greatest dimension, and nonthymomatous MG. However, skills improvement may cause an extension of indications in the future. Minimally invasive thymectomy has been associated with low postoperative morbidity and mortality (38-41). Conversion rates to open thymectomy vary in different studies from $0 \%$ to $7 \%(41-44)$.

Since the introduction of minimally invasive techniques, there have been spirited discussions regarding the optimal surgical approach. Several meta-analyses have now compared open thymectomy with minimally invasive techniques (45-48). The results indicate that minimally invasive thymectomy is safe, can achieve oncologic outcomes similar to those of open thymectomy and is associated with less morbidity and shorter length of hospital stay.

In a study of 429 patients undergoing robotic or transsternal thymectomy, 100 patients were selected for propensity-matched analysis for each group (49). Robotic thymectomy demonstrated lower incidence of postoperative complications and shorter length of stay (LOS), while longterm oncologic outcomes were comparable between the two approaches. Nevertheless, until results from prospective, randomized trials are available, the debate between open and minimally invasive thymectomy is likely to continue. Regardless of surgical approach, complete resection of a thymic tumor is considered one of the most important prognostic factors and is correlated with prolonged disease-free and overall survival (50-54). To that end, total (extended) thymectomy has been traditionally used to maximize resection of thymic tissue. This involves complete resection of the thymus with removal of all adipose tissue lying anterior to the pericardium, from the thyrothymic ligament to the diaphragm and between the phrenic nerves. A total thymectomy also includes the anterior thymic region lymph nodes (N1), as defined in the Thymic Epithelial Tumors Staging Project by the International Thymic Malignancy Interest Group, the International Association for the Study of Lung Cancer and IALSC $(55,56)$. Systemic sampling of deep thymic region lymph nodes (N2) is recommended for thymic carcinomas and for thymomas with invasion of mediastinal structures. A phrenic nerve infiltrated by the tumor can be sacrificed; however, in myasthenic patients, this approach can lead to significant respiratory compromise and is best avoided. If both phrenic nerves are involved, the surgeon must ensure preservation of at least one of those. In cases where pericardium, lung, brachiocephalic vein, superior vena cava, or chest wall are involved, the tumor is removed en bloc with the infiltrated structures.

The role of partial thymectomy in the treatment of patients with Masaoka stage I or II thymoma and without MG has been an issue of debate. Retrospective series comparing the oncologic outcomes of partial versus total thymectomy have shown no significant differences in survival, recurrence, or subsequent development of MG (57-59). However, large, prospective studies with long follow-up periods are warranted to establish partial thymectomy as a valid treatment of early-stage thymoma in non-myasthenic patients.

When surgery alone is not sufficient it is necessary a multimodal approach that integrates surgery, radiotherapy and systemic treatments.

\section{Systemic treatment}

\section{Chemotherapy}

Platinum-based chemotherapy represents the standard first line treatment for TET patients with unresectable disease or who progress after surgery and radiotherapy. The overall response rate (ORR) ranged from 50\% with cisplatin and etoposide (PE) combination, to $75 \%$ and $92 \%$ with the PAC (cisplatin, doxorubicin and cyclophosphamide) and ADOC (cisplatin, doxorubicin, cyclophosphamide and vincristine) regimens, respectively (60-62).

Carboplatin and paclitaxel regimen was evaluated in 21 thymomas and 23 thymic carcinomas $(63,64)$.

After progression to first line therapy, some patients are candidates for second-line treatment with single agent chemotherapy, such as pemetrexed or ifosfamide, achieving ORR of $20-40 \%$ (65-67). 
Table 1 Chemotherapy regimens in TETs

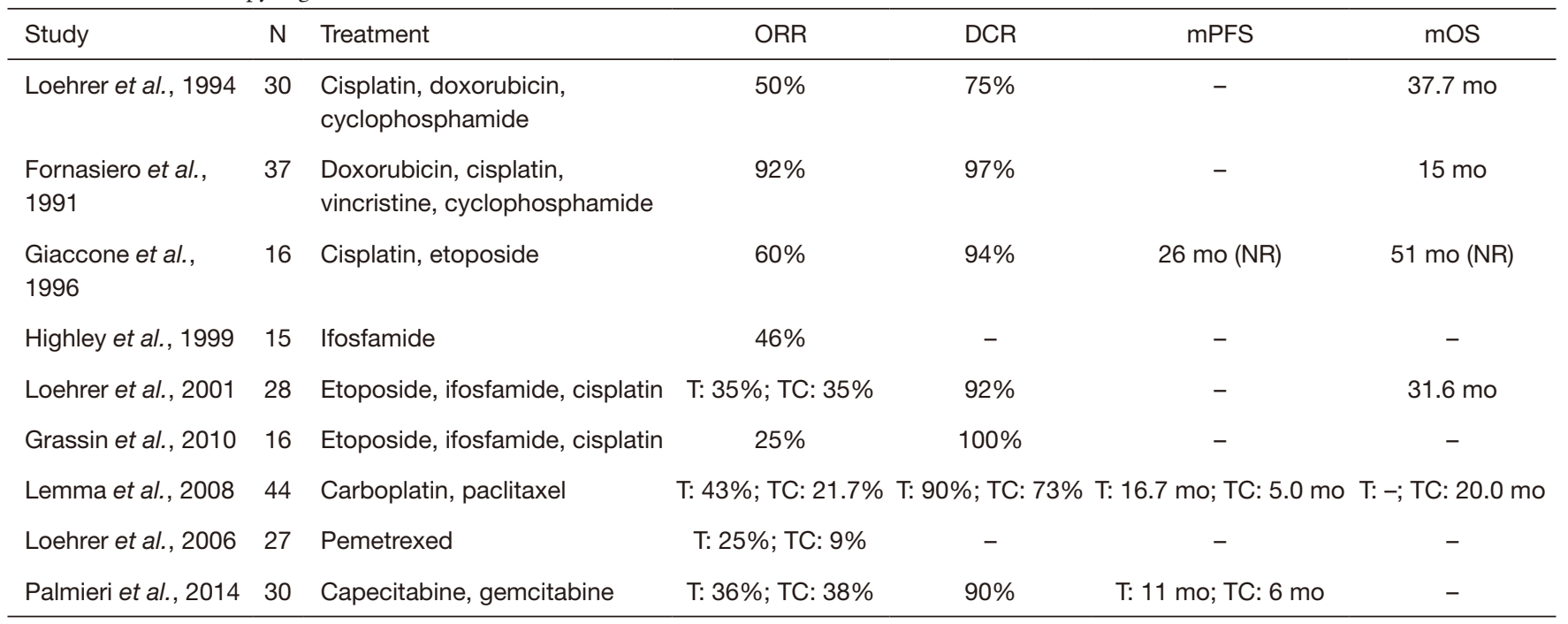

TETs, thymic epithelial tumors; ORR, overall response rate; mo, months; mPFS, median progression free survival; mOS, median overall survival; NR, not reached; T, thymoma; TC, thymic carcinoma.

The most recent study is a multicenter phase II trial to assess activity of the combination of capecitabine and gemcitabine that enrolled 12 thymomas and 3 thymic carcinomas who progressed to a first line of chemotherapy: $40 \%$ of patients achieved an objective response $(68,69)$.

Data available derive from prospective phase II trials or retrospective analyses and are summarized in Table 1 (70).

\section{New target therapies}

Some promising data have been reported on the activity of new target therapies, including anti-angiogenic drugs, cyclin dependent kinases and PI $3 \mathrm{~K} / \mathrm{mTOR}$ inhibitors and immunotherapy.

C-Kit overexpression has been reported in $50-80 \%$ of thymic carcinomas, however, it is rare in thymomas. In two small phase II trials testing the c-Kit inhibitor imatinib in unselected TETs patients, no response were reported (71-73).

Activating mutations in epidermal growth factor receptor $(E G F R)$ gene are very rare in TETs and anti EGFR drugs (i.e., gefitinib, erlotinib and cetuximab) showed very limited activity (74-76).

VEGF-A and VEGFR-1 and -2 overexpression has been described in TETs. Sunitinib, an anti-angiogenic multikinase inhibitor, was tested in patients with thymic carcinomas and thymomas. ORR was $26 \%$ in the thymic carcinoma cohort and $6 \%$ in the thymoma cohort (77). Sunitinib is currently in the NCCN guidelines for the treatment of patients with thymic carcinoma who have progressed after chemotherapy. In the phase II REMORA trial the efficacy of anti-angiogenic drugs in patients with advanced thymic carcinomas was confirmed $(78,79)$. These results suggest that Lenvatinib could become a standard treatment option for patients with previously treated advanced or metastatic thymic carcinoma. In the RELEVANT trial, a single-arm phase II study, the activity and safety of ramucirumab, an anti-VEGFR2 monoclonal antibody, combined with paclitaxel and carboplatin were tested in first line treatment of patients with thymoma B3 or thymic carcinoma (80).

Alterations of the PI3K/AKT/mTOR pathway are involved in the pathogenesis of TETs. The activity of everolimus was tested, in a single-arm phase II trial, in 50 patients with TETs, previously treated with cisplatincontaining regimens (81). The disease control rate (DCR) was $88 \%$ and the median PFS was 10 months.

The efficacy of the anti-PD-1, pembrolizumab, has been evaluated in 40 heavily pretreated patients with thymic carcinoma (82). The median follow-up was 20 months. The proportion of patients who achieved a response was $22.5 \%$. PFS of patients with high PD-L1 expression were longer than those with low or no PD-L1 expression (24vs. 2.9 months, respectively). Six (15\%) patients developed severe autoimmune toxicity, including $2(5 \%)$ patients with 
myocarditis. Another phase II trial tested pembrolizumab in 7 thymomas and 26 thymic carcinomas Asian patients, reporting ORR $28.6 \%$ in thymomas and $19.2 \%$ in thymic carcinomas (83). Also nivolumab was tested in unresectable or recurrent thymic carcinoma (84) and avelumab in advanced thymoma (85). The efficacy of these treatments should be balanced with their toxicity profiles.

A number of new drugs and combinations are currently under evaluation and we will have more results in the coming years.

\section{Radiotherapy (RT)}

Adjuvant RT is not indicated in stages I and II if there is a complete resection (assess in stages II-IV, R0, type B2 and B3). In stages III in which the resection is complete it can be debatable.

Adjuvant RT is indicated in cases of incomplete resections or positive margins, since it allows a local control of $50-80 \%$.

In adjuvant treatment, the recommended dose is 45-50 Gy after R0 resection and 50-54 Gy after R1, with an increase in areas of residual tumor (conventional fractionation: 1.8-2 Gy/day for 4-6 weeks).

In patients with an invasive tumor or a large mediastinal mass with extension to vascular structures or R2, the usual dose is around $60 \mathrm{~Gy}$.

\section{Conclusions}

Thymic tumors are relatively rare tumors with a wide spectrum of clinical presentation. In our experience, robotic assisted thoracic surgery is an excellent option for tumors up to at least $9 \mathrm{~cm}$ and without extensive invasion of surrounding structures. Nonetheless, transsternal mediastinal tumor resection still plays an important role in the treatment of advanced tumors and especially when treating thymic carcinomas. Therefore, patients should be carefully selected in order to offer the best oncological outcome possible.

\section{Acknowledgments}

This work is dedicated to the memory of Professor Dr. H.c. Herwart F. Otto (23.12.1938-23.11.2010), formerly chairman of the Institute of Pathology, of the Ruprechts Karls University of Heidelberg, Baden-Wuerttember, Germany.
Funding: None.

\section{Footnote}

Reporting Checklist: The authors have completed the Narrative Review reporting checklist. Available at https:// dx.doi.org/10.21037/gs-21-517

Conflicts of Interest: All authors have completed the ICMJE uniform disclosure form (available at https://dx.doi. org/10.21037/gs-21-517). CMS reports grants and royalties from a Springer Book. The other authors have no conflicts of interest to declare.

Ethical Statement: The authors are accountable for all aspects of the work in ensuring that questions related to the accuracy or integrity of any part of the work are appropriately investigated and resolved.

Open Access Statement: This is an Open Access article distributed in accordance with the Creative Commons Attribution-NonCommercial-NoDerivs 4.0 International License (CC BY-NC-ND 4.0), which permits the noncommercial replication and distribution of the article with the strict proviso that no changes or edits are made and the original work is properly cited (including links to both the formal publication through the relevant DOI and the license). See: https://creativecommons.org/licenses/by-ncnd/4.0/.

\section{References}

1. Engels EA. Epidemiology of thymoma and associated malignancies. J Thorac Oncol 2010;5:S260-5.

2. Minervini F, Kocher GJ. When to suspect a thymoma: clinical point of view. J Thorac Dis 2020;12:7613-8.

3. Detterbeck FC, Parsons AM. Thymic tumors. Ann Thorac Surg 2004;77:1860-9.

4. Masaoka A, Monden Y, Nakahara K, et al. Follow-up study of thymomas with special reference to their clinical stages. Cancer 1981;48:2485-92.

5. Koga K, Matsuno Y, Noguchi M, et al. A review of 79 thymomas: modification of staging system and reappraisal of conventional division into invasive and non-invasive thymoma. Pathol Int 1994;44:359-67.

6. Detterbeck FC, Stratton K, Giroux D, et al. The IASLC/ ITMIG Thymic Epithelial Tumors Staging Project: proposal for an evidence-based stage classification system 
for the forthcoming (8th) edition of the TNM classification of malignant tumors. J Thorac Oncol 2014;9:S65-72.

7. Brierley JD, Gospodarowicz MK, Wittekind C, editors. The TNM classification of malignant tumours. Oxford: Wiley Blackwell, 2017.

8. Travis WD, Brambilla E, Burke AP, et al. Introduction to The 2015 World Health Organization Classification of Tumors of the Lung, Pleura, Thymus, and Heart. J Thorac Oncol 2015;10:1240-2.

9. Coronado RA, Simon CB, Lentz TA, et al. Optimism Moderates the Influence of Pain Catastrophizing on Shoulder Pain Outcome: A Longitudinal Analysis. J Orthop Sports Phys Ther 2017;47:21-30.

10. Yajima T, Mogi A, Yamaki E, et al. Advantages of additional PET vs. MRI in the clinical diagnosis of anterior mediastinal tumors. Mol Clin Oncol 2020;13:85.

11. Benveniste MF, Moran CA, Mawlawi O, et al. FDG PETCT aids in the preoperative assessment of patients with newly diagnosed thymic epithelial malignancies. J Thorac Oncol 2013;8:502-10.

12. Su S, Colson LY. Overview of Benign and Malignant Mediastinal Diseases. In: David Sugarbaker. Adult Chest Surgery. Mc Graw Hill, 2015.

13. Santoprete S, Ragusa M, Urbani M, et al. Shock induced by spontaneous rupture of a giant thymoma. Ann Thorac Surg 2007;83:1526-8.

14. Hokka D, Ogawa H, Tane S, et al. Ruptured thymoma causing a hemothorax: A case report. Oncol Lett 2015;10:1810-2.

15. Brunner J, Boehler T, Ehemann V, et al. Decreased apoptosis despite severe CD4 depletion in the thymus of a human immunodeficiency virus-1 infected child. Klin Padiatr 2011;223:246-8.

16. Lopes DTP, Gomes SMP, Siqueira SAC, et al. Myasthenia Gravis Related to Thymic Carcinoma: A Case Study. Thorac Cardiovasc Surg Rep 2020;9:e33-6.

17. Viéitez MC, Aladró Y, Alemany MJ, et al. Myasthenia gravis associated with carcinoma of the thymus. A report of three cases. Rev Neurol 2001;32:1135-7.

18. Koneczny I, Herbst R. Myasthenia Gravis: Pathogenic Effects of Autoantibodies on Neuromuscular Architecture. Cells 2019;8:671.

19. Cavalcante P, Maggi L, Colleoni L, et al. Inflammation and epstein-barr virus infection are common features of myasthenia gravis thymus: possible roles in pathogenesis. Autoimmune Dis 2011;2011:213092.

20. Lippner EA, Lewis DB, Robinson WH, et al. Paraneoplastic and Therapy-Related Immune Complications in Thymic Malignancies. Curr Treat Options Oncol 2019;20:62.

21. Blum TG, Misch D, Kollmeier J, et al. Autoimmune disorders and paraneoplastic syndromes in thymoma. J Thorac Dis 2020;12:7571-90.

22. Dessypris EN. Pure Red Cell Aplasia. In: Shahidi NT. editor. Aplastic Anemia and Other Bone Marrow Failure Syndromes. New York: Springer, 1990.

23. Verley JM, Hollmann KH. Thymoma. A comparative study of clinical stages, histologic features, and survival in 200 cases. Cancer 1985;55:1074-86.

24. Jansen A, van Deuren M, Miller J, et al. Prognosis of Good syndrome: mortality and morbidity of thymoma associated immunodeficiency in perspective. Clin Immunol 2016;171:12-7.

25. Wadhera A, Maverakis E, Mitsiades N, et al. Thymomaassociated multiorgan autoimmunity: a graft-versus-hostlike disease. J Am Acad Dermatol 2007;57:683-9.

26. Nakagiri T, Okumura M, Inoue $M$, et al. Thymomaassociated graft-versus-host disease-like erythroderma. J Thorac Oncol 2007;2:1130-2.

27. Hayes SA, Huang J, Plodkowski AJ, et al. Preoperative computed tomography findings predict surgical resectability of thymoma. J Thorac Oncol 2014;9:1023-30.

28. Marom EM, Milito MA, Moran CA, et al. Computed tomography findings predicting invasiveness of thymoma. J Thorac Oncol 2011;6:1274-81.

29. Papatestas AE, Genkins G, Kornfeld P, et al. Transcervical thymectomy in myasthenia gravis. Surg Gynecol Obstet 1975;140:535-40.

30. Khicha SG, Kaiser LR, Shrager JB. Extended transcervical thymectomy in the treatment of myasthenia gravis. Ann N Y Acad Sci 2008;1132:336-43.

31. Calhoun RF, Ritter JH, Guthrie TJ, et al. Results of transcervical thymectomy for myasthenia gravis in 100 consecutive patients. Ann Surg 1999;230:555-9; discussion 559-61.

32. Shrager JB, Deeb ME, Mick R, et al. Transcervical thymectomy for myasthenia gravis achieves results comparable to thymectomy by sternotomy. Ann Thorac Surg 2002;74:320-6; discussion 326-7.

33. de Perrot M, Bril V, McRae K, et al. Impact of minimally invasive trans-cervical thymectomy on outcome in patients with myasthenia gravis. Eur J Cardiothorac Surg 2003;24:677-83.

34. Bril V, Kojic J, Ilse WK, et al. Long-term clinical outcome after transcervical thymectomy for myasthenia gravis. Ann 
Thorac Surg 1998;65:1520-2.

35. Yano M, Moriyama S, Haneda H, et al. Thymectomy using the subxiphoid approach. J Thorac Cardiovasc Surg 2016;152:278-9.

36. Li J, Qi G, Liu Y, et al. Meta-analysis of subxiphoid approach versus lateral approach for thoracoscopic Thymectomy. J Cardiothorac Surg 2020;15:89.

37. Pinzon D, Byrns S, Zheng B. Prevailing Trends in Haptic Feedback Simulation for Minimally Invasive Surgery. Surg Innov 2016;23:415-21.

38. Meacci E, Cesario A, Margaritora S, et al. Thymectomy in myasthenia gravis via original video-assisted inframammary cosmetic incision and median sternotomy: longterm results in 180 patients. Eur J Cardiothorac Surg 2009;35:1063-9; discussion 1069.

39. Meyer DM, Herbert MA, Sobhani NC, et al. Comparative clinical outcomes of thymectomy for myasthenia gravis performed by extended transsternal and minimally invasive approaches. Ann Thorac Surg 2009;87:385-90; discussion 390-1.

40. Pompeo E, Tacconi F, Massa R, et al. Long-term outcome of thoracoscopic ex-tended thymectomy for nonthymomatous myasthenia gravis. Eur J Cardiothorac Surg 2009;36:164-9.

41. Marulli G, Rea F, Melfi F, et al. Robot-aided thoracoscopic thymectomy for ear-ly-stage thymoma: a multicenter European study. J Thorac Cardiovasc Surg 2012;144:112530.

42. Cheng YJ, Kao EL, Chou SH. Videothoracoscopic resection of stage II thymoma: prospective comparison of the results between thoracoscopy and open methods. Chest 2005;128:3010-2.

43. Rückert JC, Swierzy M, Ismail M. Comparison of robotic and nonrobotic thoracoscopic thymectomy: a cohort study. J Thorac Cardiovasc Surg 2011;141:673-7.

44. Jurado J, Javidfar J, Newmark A, et al. Minimally invasive thymectomy and open thymectomy: outcome analysis of 263 patients. Ann Thorac Surg 2012;94:974-81; discussion 981-2.

45. Friedant AJ, Handorf EA, Su S, et al. Minimally Invasive versus Open Thymectomy for Thymic Malignancies: Systematic Review and Meta-Analysis. J Thorac Oncol 2016;11:30-8.

46. Gung Y, Zhang H, Li S, et al. Sternotomy versus videoassisted thoracoscopic surgery for thymectomy of myasthenia gravis patients: A meta-analysis. Asian J Endosc Surg 2016;9:285-94.
47. Qi K, Wang B, Wang B, et al. Video-assisted thoracoscopic surgery thymectomy versus open thymectomy in patients with myasthenia gravis: a meta-analysis. Acta Chir Belg 2016;116:282-8.

48. Yang Y, Dong J, Huang Y. Thoracoscopic thymectomy versus open thymectomy for the treatment of thymoma: A meta-analysis. Eur J Surg Oncol 2016;42:1720-8.

49. Kang CH, Hwang Y, Lee HJ, et al. Robotic thymectomy in anterior mediastinal mass: propensity score matching study with transsternal thymectomy. Ann Thorac Surg 2016;102:895-901.

50. Regnard JF, Magdeleinat P, Dromer C, et al. Prognostic factors and long-term results after thymoma resection: a series of 307 patients. J Thorac Cardiovasc Surg 1996;112:376-84.

51. Nakagawa K, Asamura H, Matsuno Y, et al. Thymoma: a clinicopathologic study based on the new World Health Organization classification. J Thorac Cardiovasc Surg 2003;126:1134-40.

52. Rea F, Marulli G, Girardi R, et al. Long-term survival and prognostic factors in thymic epithelial tumours. Eur J Cardiothorac Surg 2004;26:412-8.

53. Weis CA, Yao X, Deng Y, et al. The impact of thymoma histotype on prognosis in a worldwide database. J Thorac Oncol 2015;10:367-72.

54. Corona-Cruz JF, López-Saucedo RA, Ramírez-Tirado LA, et al. Extended resections of large thymomas: importance of en bloc thymectomy. J Thorac Dis 2018;10:3473-81.

55. Bhora FY, Chen DJ, Detterbeck FC, et al. The ITMIG/ IASLC Thymic Epithelial Tumors Staging Project: A Proposed Lymph Node Map for Thymic Epithelial Tumors in the Forthcoming 8th Edition of the TNM Classification of Malignant Tumors. J Thorac Oncol 2014;9:S88-96.

56. Ruffini E, Fang W, Guerrera F, et al. The International Association for the Study of Lung Cancer Thymic Tumors Staging Project: The Impact of the Eighth Edition of the Union for International Cancer Control and American Joint Committee on Cancer TNM Stage Classification of Thymic Tumors. J Thorac Oncol 2020;15:436-47.

57. Onuki T, Ishikawa S, Iguchi K, et al. Limited thymectomy for stage I or II thymomas. Lung Cancer 2010;68:460-5.

58. Tseng YC, Hsieh CC, Huang HY, et al. Is thymectomy necessary in nonmyasthenic patients with early thymoma? J Thorac Oncol 2013;8:952-8. 
59. Nakagawa K, Asamura H, Sakurai H, et al. Does the mode of surgical resection affect the prognosis/recurrence in patients with thymoma? J Surg Oncol 2014;109:179-83.

60. Loehrer PJ Sr, Kim K, Aisner SC, et al. Cisplatin plus doxorubicin plus cyclophosphamide in metastatic or recurrent thymoma: final results of an intergroup trial. The Eastern Cooperative Oncology Group, Southwest Oncology Group, and Southeastern Cancer Study Group. J Clin Oncol 1994;12:1164-8.

61. Fornasiero A, Daniele O, Ghiotto C, et al. Chemotherapy for invasive thymoma. A 13-year experience. Cancer 1991;68:30-3.

62. Giaccone G, Ardizzoni A, Kirkpatrick A, et al. Cisplatin and etoposide combination chemotherapy for locally advanced or metastatic thymoma. A phase II study of the European Organization for Research and Treatment of Cancer Lung Cancer Cooperative Group. J Clin Oncol 1996;14:814-20.

63. Lemma GL, Lee JW, Aisner SC, et al. Phase II study of carboplatin and paclitaxel in advanced thymoma and thymic carcinoma. J Clin Oncol 2011;29:2060-5.

64. Okuma Y, Saito M, Hosomi Y, et al. Key components of chemotherapy for thymic malignancies: a systematic review and pooled analysis for anthracycline-, carboplatinor cisplatin-based chemotherapy. J Cancer Res Clin Oncol 2015;141:323-31.

65. Gbolahan OB, Porter RF, Salter JT, et al. A Phase II Study of Pemetrexed in Patients with Recurrent Thymoma and Thymic Carcinoma. J Thorac Oncol 2018;13:1940-8.

66. Loehrer PJ, Yiannoutsos CT, Dropcho S, et al. A phase II trial of pemetrexed in patients with recurrent thymoma or thymic carcinoma. J Clin Oncol 2006;24:abstr 7079.

67. Highley MS, Underhill CR, Parnis FX, et al. Treatment of invasive thymoma with single-agent ifosfamide. J Clin Oncol 1999;17:2737-44.

68. Palmieri G, Merola G, Federico P, et al. Preliminary results of phase II study of capecitabine and gemcitabine (CAP-GEM) in patients with metastatic pretreated thymic epithelial tumors (TETs). Ann Oncol 2010;21:1168-72.

69. Palmieri G, Buonerba C, Ottaviano M, et al. Capecitabine plus gemcitabine in thymic epithelial tumors: final analysis of a Phase II trial. Future Oncol 2014;10:2141-7.

70. Conforti F, Pala L, Giaccone G, et al. Thymic epithelial tumors: From biology to treatment. Cancer Treat Rev 2020;86:102014.

71. Giaccone G, Rajan A, Ruijter R, et al. Imatinib mesylate in patients with WHO B3 thymomas and thymic carcinomas. J Thorac Oncol 2009;4:1270-3.

72. Salter JT, Lewis D, Yiannoutsos PJ, et al. Imatinib for the treatment of thymic carcinoma. J Clin Oncol 2008;26:abstr 8116.

73. Ströbel P, Hartmann M, Jakob A, et al. Thymic carcinoma with overexpression of mutated KIT and the response to imatinib. N Engl J Med 2004;350:2625-6.

74. Bedano PM, Perkins S, Burns M, et al. A phase II trial of erlotinib plus bevacizumab in patients with recurrent thymoma or thymic carcinoma. J Clin Oncol 2008;26:abstr 19087.

75. Farina G, Garassino MC, Gambacorta M, et al. Response of thymoma to cetuximab. Lancet Oncol 2007;8:449-50.

76. Palmieri G, Marino M, Salvatore M, et al. Cetuximab is an active treatment of metastatic and chemorefractory thymoma. Front Biosci 2007;12:757-61.

77. Thomas A, Rajan A, Berman A, et al. Sunitinib in patients with chemotherapy-refractory thymoma and thymic carcinoma: an open-label phase 2 trial. Lancet Oncol 2015;16:177-86.

78. Itoh S, Satouchi M, Sato J, et al. Durable anti-tumor activity of the multi-targeted inhibitor lenvatinib in patients with advanced or metastatic thymic carcinoma; Preliminary results from a multicenter phase II (REMORA) trial. Ann Oncol 2019;30:v747-55.

79. Sato J, Satouchi M, Itoh S, et al. Lenvatinib in patients with advanced or meta-static thymic carcinoma (REMORA): a multicentre, phase 2 trial. Lancet Oncol 2020;21:843-50.

80. Imbimbo M, Vitali M, Fabbri A, et al. RELEVENT Trial: Phase II Trial of Ramucirumab, Carboplatin, and Paclitaxel in Previously Untreated Thymic Carcinoma/B3 Thymoma With Area of Carcinoma. Clin Lung Cancer 2018;19:e811-4.

81. Zucali PA, De Pas T, Palmieri G, et al. Phase II Study of Everolimus in Patients With Thymoma and Thymic Carcinoma Previously Treated With Cisplatin-Based Chemotherapy. J Clin Oncol 2018;36:342-9.

82. Giaccone G, Kim C, Thompson J, et al. Pembrolizumab in patients with thymic carcinoma: a single-arm, singlecentre, phase 2 study. Lancet Oncol 2018;19:347-55.

83. Cho J, Kim HS, Ku BM, et al. Pembrolizumab for Patients With Refractory or Relapsed Thymic Epithelial Tumor: An Open-Label Phase II Trial. J Clin Oncol 2019;37:2162-70.

84. Katsuya Y, Horinouchi H, Seto T, et al. Single-arm, 
multicentre, phase II trial of nivolumab for unresectable or recurrent thymic carcinoma: PRIMER study. Eur J Cancer 2019;113:78-86.

85. Rajan A, Heery CR, Thomas A, et al. Efficacy and

Cite this article as: Minervini F, Boschetti L, Gregor M, Provencio M, Calvo V, Kestenholz PB, Lampridis S, Patrini D, Bertoglio P, Azenha LF, Sergi CM, Kocher GJ. Thymic tumours: a single center surgical experience and literature review on the current diagnosis and management of thymic malignancies. Gland Surg 2021;10(11):3128-3140. doi: $10.21037 / g s-21-517$ tolerability of anti-programmed death-ligand 1 (PD-L1) antibody (Avelumab) treatment in advanced thymoma. J Immunother Cancer 2019;7:269. 